\section{L’approccio unitario alla malattia renale garantisce cure migliori}

\section{Caro Direttore,}

ho letto con estremo interesse la tua lettera e ne condivido totalmente $\mathrm{i}$ fondamenti. Si inserisce in un potenziale percorso di analisi e proposte finalizzate a sostenere l'attività specialistica del nefrologo e a evitare la tendenza alla parcellizzazione delle competenze. L'approccio alla malattia renale deve avere un carattere complessivo e unitario. II nefrologo è il medico specialista di riferimento e non il tecnico della dialisi. Purtroppo con le normative di riordino delle reti sanitarie di cura (Decreto Balduzzi, DM 70), il riferimento della malattia renale non è il fenomeno pandemico assunto dalla patologia anche in Italia, ma i 55 mila dializzati. Vi è anche un contrasto tra elaborati programmatici, dato che nel Piano nazionale delle malattie croniche, la malattia renale (una delle sei individuate dal Piano) viene considerata invece in modo unitario: fase di prevenzione, uremia terminale, trapianto, follow-up post-trapianto. Su tutto spicca il principio di una presa in carico del paziente a 360 gradi. Lo strumento indicato è il PDTA che dovrebbe, secondo il mio parere, comprendere la questione degli accessi vascolari e le forme cliniche e organizzative che vi devono presiedere. La proposta della presenza del nefrologo in questo senso è assolutamente da condividere e da rendere esigibile.

\section{Disclosures}

Financial support: No financial support was received for this submission.

Conflict of interest: The author has no conflict of interest.

\section{Giuseppe Vanacore}

Presidente dell'Associazione Nazionale Emodializzati - Dialisi e Trapianto Onlus, Milano

Indirizzo per la corrispondenza:

Dr. Giuseppe Vanacore

Presidente dell'Associazione Nazionale Emodializzati - Dialisi

e Trapianto Onlus

Via Hoepli 3

20121 Milano

presidente@aned-onlus.it

Published online: May 3, 2017 